\title{
New Results in Discrete-Time Loop Transfer Recovery
}

\section{Niemann, Hans Henrik; Søgaard-Andersen, Per}

\section{Published in:}

American Control Conference

Publication date:

1988

\section{Document Version}

Publisher's PDF, also known as Version of record

Link back to DTU Orbit

Citation (APA):

Niemann, H. H., \& Søgaard-Andersen, P. (1988). New Results in Discrete-Time Loop Transfer Recovery. In American Control Conference (pp. 2483-2489). IEEE.

\section{General rights}

Copyright and moral rights for the publications made accessible in the public portal are retained by the authors and/or other copyright owners and it is a condition of accessing publications that users recognise and abide by the legal requirements associated with these rights.

- Users may download and print one copy of any publication from the public portal for the purpose of private study or research.

- You may not further distribute the material or use it for any profit-making activity or commercial gain

- You may freely distribute the URL identifying the publication in the public portal

If you believe that this document breaches copyright please contact us providing details, and we will remove access to the work immediately and investigate your claim 


\section{MEN RESUTS IN DISCRETE-TIKE LOOP IRAMSER RECOVERY}

Hans Henrik Niemann and Per SFgaard-Andersen

Control Engineering Institute, Technical University of Denmark, Building 424, OK-2800 Lyngby, Denmark.

\begin{abstract}
For discrete-time compensators incorporating prediction observers asymptotic loop transfer recovery is not feasible. Instead loop transfer recovery objectives must be satisfied via exact recovery techniques. In this note the modelbased compensators which achieves exact recovery are parametrized in terms of the system zeros and the corresponding zero-directions. Fullorder as well as minimal-order observers are treated. Further it is shown how exact recovery is also applicable to non-minimum phase plants. In this case the achievable performance is parameterized explicitly.
\end{abstract}

\section{INTROTICTION}

In recent years the LOG/LTR feedback design methodology for robust model-based compensation has received much attention [see o.g. 1-6]. This procedure works for continuous-time systems and it is always effective for minimum-phase plants. Unfortunately a similar procedure is not generally feasible in discrete-time. If filtering observers are used asymptotic recovery (the LTR step) is often possible [11]. However, the application of filtering observers require that the processing time of computing the control signal is negligible in comparison to the sampling interval. Very often such an assumption cannot be satisfied in practice, and prediction observers must be used. For compensators based on prediction observers, however. the asymptotic procedures will not be effective, since in general the difference between a fullstate loop transfer (target design) and the full asymptotic loop transfer remains finite [4,11]. A detailed discussion of the mechanisms behind this fact is given in [4].

Loop transfer recovery is still possible, however, but different methods must be applied. In [6,11] such methods are discussed - and referred to as exact loop transfer recovery. In [4] the conditions for exact recovery for fullorder observers were outlined, and some preliminary design considerations for minimumphase continuous-time systems based on fullorder observers were presented in [10]. In this note a more general treatment of exact recovery in discrete-time is provided. Exact recovery for minimum-phase as well as non-minimum phase plants based on full-order observers are discussed. Further results on exact recovery based on minimal-order observers are presented, and it is shown that in certain - common - cases very powerful designs procedures are possible. This is the first treatment of LTR for minimalorder observers in discrete-time. Earlier studies $[16,17]$ were in continuous-time, but due to the same problems as for full-order observers the continuous-time methods cannot be generalized to discrete-time. Hence new methods based on exact recovery must be developed. Notice that the issue of recovery for non-minimum phase is particulary relevant in discrete-time since the sampling proces often produces zeros outside the unit-circle [13]. An advantage of using the exact recovery concepts presented here is that the controllers are of finite gains, whereas the usual continuous-time LQG/LTR method often produces high-gain controllers.

The paper is organized as follows. In $\$ 2-4$ the full-order observer case is treated, and in 5 5-7 minimal-order observer results are presented follow in 58 by some examples.

\section{EXACT LOOP TRAUSFER RECOVERY}

In the following square discrete-time minimum phase systems $S(A, B, C)$ are considered. It will be assumed that the model is minimal. The plant $t r a n s f e r$ matrix $G(z)$ and the model-based compensator $H(z)$ are given

$$
\begin{array}{rlrl}
G(z) & =C \not(z) B, & \operatorname{dim} G(z) & =m \times m \\
\phi(z) & =(z I-A)^{-1} & \operatorname{dim} \phi(z) & =n \times n \\
H(z)=K(z I-A+B K+F C)^{-1} F, \operatorname{dim} H(z) & =m \times m \\
& & \mid 2-1)
\end{array}
$$

Here $K$ is the full-state feedback gain and $F$ is the full-order observer-gain. Let the number of transmission zeros be $p$. In order to formulate the loop-shape robustness constraints the uncertainties (disturbances, noise and modelling errors) are reflected to the plant input mode $[4,14]$. The target loop transfer is then the full-state loop transfer $K \gg B$ and the full loop transfer is HG [3,5]. The difference between these two indicators is defined as the loop recovery error $E_{I}(z)$ :

$$
E_{I}(z)=K \phi(z) B-H(z) G(z)
$$

In order to have exact recovery it is required that $E_{1}(z) \equiv 0$ for all $z$. For square systems Goodman [4] has shown that

$$
\begin{aligned}
& E_{I}(z)=M_{I}(z)\left(I+M_{I}(z)\right)^{-1}(I+K \uparrow(z) \theta) \\
& M_{I}(z)=K(z I-A+F C)^{-1} B
\end{aligned}
$$


It is, however, straightforward to derive the same results for non-square systems as well. Now let $M_{I}(z)$ be rewritten in the residual form:

$$
H_{I}(z)=\sum_{i=1}^{n} \frac{k v_{i} w_{i}^{T} B}{z-\lambda_{i}}
$$

where $v_{j}$ and $w_{i}^{T}$ are right and left eigenvectors associated with the oigenvalue $\lambda_{i}$ of $A-F C$. It is easy to show that

$$
\begin{aligned}
& E_{I}(z)=0 \quad \text { iff } \\
& H_{I}(z)=0 \text { iff } \quad(2-5 a, b, c) \\
& K v_{i}=0 \text { or } w_{i}^{\top}=0, i=1, \ldots, n
\end{aligned}
$$

if $A-F C$ is non-defective. The latter foralation of the exact recovery condition is suitable deriving the associated compensators.

\section{3 solutar of THE EXAT LTR PROCEN}

From oigenstructure assignment it is known that the left eigenvectors $w_{i}$ with the eigenvalue $\lambda_{i}$ of $A-F C$ are given by $\{g\}$ :

$$
\begin{aligned}
& {\left[\begin{array}{ll}
w^{\top}{ }_{i} & z_{i}^{\top}
\end{array}\right]\left[\begin{array}{c}
\lambda_{i} I-A \\
-c
\end{array}\right]=0, i=1, \ldots, n} \\
& w_{i}^{\top} F=-z_{i}^{\top}
\end{aligned}
$$

The condition $w_{i}^{T}=0$ from $(2-5)$ imply that

$$
\left[\begin{array}{ll}
w_{i 0}^{\top} z_{i 0}^{\top}
\end{array}\right]\left[\begin{array}{cc}
\lambda_{i 0}^{I}-A & B \\
-c & 0
\end{array}\right]=0
$$

Maximally $p$ eigenvectors $w_{j 0}^{\top}$ can satisfy this candition, if $\lambda_{j}$ is select:d as transmission zero of $S\left(A, B, C f^{0}[B]\right.$ : Let these $p$ eigenvalues $/$ vectors be selected from $(3-2)$, it is then straightforward to see that $F$ is parameterized by:

$$
\begin{aligned}
& F=\left[\begin{array}{c}
w_{1}^{\top} \\
\vdots \\
w_{n}^{\top}
\end{array}\right]^{-1}\left[\begin{array}{c}
z_{1}^{\top} \\
\vdots \\
z_{n}^{\top}
\end{array}\right] \\
& z_{i}^{\top}=z_{i 0}^{\top}, w_{i}^{\top} \cdot w_{i 0}^{\top} i=1 \ldots, p
\end{aligned}
$$

and $\left(\lambda_{i}, z_{i}^{\top}, i=p+1, \ldots, \ldots, n\right)$ are tree design parameters. since $w_{i}^{+}$is determined by $\lambda_{i}$ and 2 . The remaining $n-p$ conditions in $(2-5 c)$ aust be satisfied by selecting $k$ suitably. Condition (2-5c) inply

$$
x\left[v_{1}, \ldots \ldots v_{n}\right]=\left[\begin{array}{ll}
0 & 0
\end{array}\right]
$$

with dim $Q=m \times p$ but otherwise arbitrary. Now

$$
K=00 \log ^{-1}=0\left[\begin{array}{c}
w_{10}^{\top} \\
\vdots \\
w_{p 0}^{\top}
\end{array}\right]=0 r
$$

with dim $r=p_{1} \times n$. $r$ consists of the left eigenvectors $w_{i p}^{\top}$ comstrained in $(3-2)$, and is thus a matrix of fixed elements. Eq (3-3) and (3-5) are therefore simple parameterizations of the controller matrices which achieves exact recovery.

A few important consequences of exact LTR are discussed next:

* The parameterization of the state-feedback imply that $K$ must be selected as an output feedback controller, where $Q$ is the free parameter output feedback matrix. $\Gamma$ is the equivalent output matrix with $p$ independent colums. Since of $n-m, a=m+p-1 \quad(<n)$ eigenvalues can be assigned for such a problem [7]. Consequently all of the close-10op eigenvalues cannot be assigned freely, and no stability guarantees are available. However in square discrete-time systems the rank[CB] is often maximal. This ensures that $6(2)$ has the maximum possible number of finite zeros. Which in turn will result in maximal freedom in selection of $k$.

* The selection of $F$ is only constrained by $e q$. (3-3) and stability can always be achived.

- Good input sensitivity and stability for plant input modelling errors can only be achived if p>m. If rank[K]< $(p<m)$ the target loop transfer $K \otimes B$ is rank defective and loopshaping is not feasible.

* Dual results apply for the plant output $200 p$ breaking point.

* The structure of the controller $H(z)$ can be studied by looking at the system matrix for the controller $P_{H}$ :

$$
P_{H}=\left[\begin{array}{cc}
I Z-A+B K+F C & F \\
K & 0
\end{array}\right]
$$

Oy using the transformation atrix $T=$ diag $\left(v^{-1}, 1\right)$ i. $\left.09.13-6\right)$ can be transformed into:

$$
P_{H}=\left[\begin{array}{ccr}
1 z-A_{D} & 0 & -z_{1} \\
\gamma Q & 1 z-\lambda_{n-p} & -z_{2} \\
0 & 0 & 0
\end{array}\right]
$$

where $y, Z$ and $Z$, has full rank. $A$ are the plant zeros and $\Lambda^{2}$ are the remaining $n-p$ poles of $A$ - FC asfigned in eq. $(3-3)$. Notice that $A$ are the poles of $H(z)$ and $A$ are output decoupling zeros of $H(z)$. Hence the resulting loop transfer $H 6$ will have $n$ poles.

* It has been assumed that $S(A, B, C)$ is minimal. The results could be extended to non-mimimal systens as well - although this issue is not pursued here.

* Further the treatient is also possible for non-square systems. Since this is strainhtforward no dotails are given here.

* Notice that the exact recovery controllers outlined above are of finite gains, whereas the continuous-time LOG/LTR procedures usually produces a high-gain controller.

\section{MON-HINIMLA-PHSE SYSTES}

sampling of a continuous-time system will often result in a non-minimum phase discrete-time 
system [13]. If the LTR results from section 3 are used on a non-minimum phase system $G(z)$, the resulting controller will be unstable. It is, however, still possible to achieve LTR for non-minimum phase systems. In order to facilitate exact recovery for non-minimum phase plants note, that in selecting $F$ only a subset $j$ of the eigenvectors constrained by eq. (3-2) need to be chosen. In doing this, however, the dimension of $Q$, the free parameters of $K$, is reduced to $m \times j$. Consequently such selection are only advisible for non-minimum phase systens. If only the plant's q minimum phase zeros are used in eq. (3-2), the equations for $F$ and $K$ become:

$$
\begin{aligned}
F & =-\left[\begin{array}{c}
w_{1}^{\top} \\
\vdots \\
w_{n}^{\top}
\end{array}\right]^{-1}\left[\begin{array}{l}
z_{1}^{\top} \\
\vdots \\
z_{n}^{\top}
\end{array}\right] \\
z_{i}^{\top} & =z_{i 0}^{\top}, w_{i}^{\top}=w_{i 0}^{\top}, i=1, \ldots, q \\
k & =\bar{q}\left[\begin{array}{c}
w_{10}^{\top} \\
w_{q 0}^{\top}
\end{array}\right] \\
& =\bar{q}
\end{aligned}
$$

where $\operatorname{dim} \bar{Q}=m \times q$.

Some of the consequences of exact LTR for nonminimum phase plants are:

* The following equation will be satisfied

$$
K \phi(z) B=H(z) G(z)
$$

The non-minimum zeros of $G$ are not cancelled out on the right hand side. Hence $H G$ and $K \$ B$ are both non-minimum phase. This in turn limits the achievable performance [12], and "good" loop-shapes for K\$B are, of course. difficult to achive. Notice how the achievable 100p-shapes - under the exact recovery constraint - are parameterized explicitly in eq. $(4-1)$ by the constraints of $K$. This results is in agreement with the results in [18].

* The freedom in the selection of $K$ will decrease by the number of non-minimum phase zeros in $G(z)$.

* The consequences of exact LTR from section 3 are still valid.

\section{MINIML ORLER OBSERVERS}

In the following the discrete-time system $S(A, B, C)$ will be partitioned as:

$$
\begin{aligned}
& A=\left[\begin{array}{ll}
A_{11} & A_{12} \\
A_{21} & A_{22}
\end{array} \underset{m}{\stackrel{n}{\longrightarrow}} \prod_{n-m}^{m} \quad B=\left[\begin{array}{l}
B_{1} \\
B_{2}
\end{array}\right] \prod^{m}\right.
\end{aligned}
$$

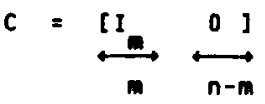

There is no loss of generality in assuming that $C=\left[I_{m} 0\right]$ since any system can be transformed into this form. The system is assumery to ot minimum-phase, with p zeros. The minimal order observer for $(5-1)$ is [15]:

$$
\begin{aligned}
& z(k+1)=O z(k)-G u(k)+E y(k) \\
& x(k)=\left[\begin{array}{l}
x_{1}(k) \\
x_{2}(k)
\end{array}\right]=\left[\begin{array}{ll}
I & 0 \\
v_{2} & I_{n-m}
\end{array}\right]\left[\begin{array}{l}
y(k) \\
z(k)
\end{array}\right]
\end{aligned}
$$

with

$$
\begin{aligned}
D & =A_{22}-V_{2} A_{12} \\
G & =B_{2}-V_{2}^{B} 1 \\
E & =A_{12}-V_{2} A_{11}+A_{22} V_{2}-V_{2} A_{12} V_{2}
\end{aligned}
$$

and $v_{2}$ is the observer gain matrix.

The feedback law is:

$u(k)=-k x(k)=-k_{1} x_{1}(k)-k_{2} x_{2}(k)$

It is assumed that $(C, A)$ is observable, which implies that $\left(A_{12}, A_{22}\right)$ is observable $[15]$.

It is known that the separation principle applies for this feedback system. Hence stability is achieved by making the full-state and the minimal-order observer stable. The condition for LTR for the minimal-order observer based design is $[16,17]$ :

$$
V_{2}\left(I+A_{12} \phi_{22} V_{2}\right)^{-1} A_{12} \phi_{22}\left(B_{2}-V_{2} B_{1}\right)=B_{2}-V_{2} B_{1}
$$

where

$$
\uparrow_{22}=\left(12-A_{22}\right)^{-1}
$$

This condition is similar to the continuous-time version, but the design results from $[16,17]$ can not be generalized, and new methods for utilising $(5-4)$ in discrete-tine are derived in $\$ 6$. If $(5-4)$ is satisfied then:

$$
K_{2}\left(t_{22}^{-1}+V_{2} A_{12}\right)^{-1}\left(B_{2}-V_{2} B_{1}\right)=0
$$

is also satisfied $[16,17]$. Eq. $(5-5)$ is a necessary and sufficient condition for LTR with minimal-order observers. If $(5-4)$ is satisfied the full-state loop transfer $K \$ B$ and the minimal-order observer based loop transfer are identical.

Let (5-5) be rewitten in the residual form:

$$
0=\sum_{i=1}^{n-m} \frac{k_{2} v_{i} w_{i}^{\top}\left(B_{2}-v_{2} B_{1}\right)}{z-\lambda_{i}}
$$

where $v_{i}$ and $w_{i}^{\top}$ are right and left eigenvectors associated with the eigenvalue $\lambda_{i}$ of $A_{22}-V_{2} A_{12}$ and from eigenstructure assignment easily found that

$$
\begin{aligned}
& w_{i}^{T}=z_{i}^{\top} \wedge_{12}{ }^{\top}{ }^{2}\left(\lambda_{i}\right), i=1, \ldots, n-m(5-7) \\
& w_{i}^{T} v_{2}=-z_{i}^{T}
\end{aligned}
$$


It is easy to show that eq. $(5-6)$ is satisfied if:

$$
k_{2} v_{i}=0 \text { or } w_{i}^{T}\left(B_{2}-v_{2} \theta_{1}\right)=0, i=1, \ldots, n-m
$$

The condition implies 3 different design cases depending on the rank of $B$.

\section{LTR SOUTIONS FOR MINIMN OAIER GaSERVES}

Case $1 \quad r\left(8_{1}\right)=0$

The recovery condition $(5-9)$ now becomes:

$$
x_{2} v_{i}=0 \text { or } \quad w_{i}^{\top} B_{2}=0, i=1, \ldots, n-m(6-1)
$$

The second condition in $(6-1)$ together with (5-7) result in:

$$
z_{10}^{T} A_{12}{ }_{22}\left(A_{10}\right) B_{2}=0,1=1, \ldots, n-m
$$

This condition can be satisfied if $\lambda$ is selected as the transmission zeros of $s(A, 8, C)$. see [6]. Eq. $(6-2)$ can be satisfied for aximally $p$ eigenvalues $\lambda$, [0]. Let these -igenvalues be selected fign $(6-2)$, it is then straightforward to see that $v_{2}$ is parameterized by:

$$
\begin{aligned}
& v_{2}=-\left[\begin{array}{c}
w_{1}^{\top} \\
\vdots \\
w_{n-m}^{\top}
\end{array}\right]^{-1}\left[\begin{array}{c}
z_{1}^{\top} \\
\vdots \\
z_{n-m}^{\top}
\end{array}\right]=-w^{\top} z \\
& z_{i}^{T}=z_{i 0}^{T}, w_{i}^{T}=z_{i 0^{\wedge}}^{\top}{ }_{12}{ }_{22}\left(\lambda_{i 0}\right), i=1, \ldots, p
\end{aligned}
$$

and $A_{i}, z_{i}^{T}, i=p+1, \ldots, n-m$ are free design paranters. The first equation in $(6-1)$ aust be satisfied for the remaining $n-n-p$ conditions by selecting $K_{2}$ as:

$$
k_{2}\left[v_{1} \ldots \ldots v_{n-n}\right]=\left[\begin{array}{lll}
2 & 0
\end{array}\right]
$$

with dim $Q=x$ put otherwise arbitrary. Now

$$
\begin{aligned}
k_{2} & =\left[a \left[v^{-1}\right.\right. \\
& =0\left[\begin{array}{c}
w_{10}^{T} \\
\vdots \\
\omega_{p}^{T}
\end{array}\right]=0 r
\end{aligned}
$$

with din $r=p \times(n-m)$. $r$ consists of the left eigenvectors $w_{i 0}^{T}$ constrained in $(6-2)$.

Gere. $\quad\left(B_{1}\right)=$

The condition $r\left(B_{1}\right)=m$ indicate that the systew $S(A, B, C)$ has $p=n-m$ zeros. The recovery conditions can now be satisfied only oy $v_{2}$ and $K_{2}$ is free to design.

The recovery condition is:

$$
w_{i}^{T}\left(B_{2}-v_{2} B_{1}\right)=0, i=1, \ldots \ldots n-n
$$

This equation can be rewritten as (by using eqs. $(5-7)$ and $(5-8))$ :

$$
z_{i 0}^{T}\left(A_{12} \phi_{22}\left(\lambda_{i 0}\right) B_{2}+\theta_{1}\right)=0
$$

The $n-m$ equations can be satisfied by selecting $\lambda_{i 0}$ as the zeros of the system $S(A, B, C)$, see [6]. The solution is:

$$
v_{2}=-\left[\begin{array}{c}
w_{1}^{\top} \\
\vdots \\
w_{n-m}^{T}
\end{array}\right]^{-1}\left[\begin{array}{c}
z_{1}^{T} \\
\vdots \\
z_{n-m}^{T}
\end{array}\right]^{\top}=-w^{-1} z
$$

with $w_{i}^{T}=z_{i 0}^{T} A_{12}{ }_{22}\left(\lambda_{i 0}\right)$ and $z_{i}^{T}=z_{10}^{T}$

$i=1, \ldots, n-m$

Notice that $V_{\text {, }}$ is uniquely determined. A different expression can be derived from (6-6):

$$
v_{2}=B_{2} B_{1}^{-1}
$$

Case 3. $0<x(8,1)<$

The recovery condition $(5-9)$ is:

$$
K_{2} v_{i}=0 \text { or } w_{i}^{\top}\left(B_{2}-v_{2} B_{1}\right)=0, i=1, \ldots, n-n
$$

The second recovery condition can again be rewritten as:

$$
2_{10}^{T}\left(A_{12} \phi_{22}\left(A_{10}\right) \theta_{2}+B_{1}\right)=0
$$

Maximally $p, p<n-m$, eigenvectors $w_{\text {io }}^{\top}$ satisfy this condition by selecting the eigenvalues $\lambda_{i}=$ $\lambda_{j}$ as the zeros of the system $S(A, B, C)$, and $z_{i}^{T}=$ $z$ as the corresponding zerodirections 1 see [b]). The first equation in $(6-10)$ must then satisfy the rewaining $n-m-p$ conditions by suitably selecting $K_{2}$.

The solution in this case is similar to case 1 .

$$
v_{2}=-\left[\begin{array}{c}
w_{1}^{\top} \\
\vdots \\
w_{n-m}^{\top}
\end{array}\right]^{-1}\left[\begin{array}{c}
z_{1}^{\top} \\
\vdots \\
z_{n-m}^{\top}
\end{array}\right]=-w^{-1} 2
$$

with $z_{i}^{T}=z_{i 0}^{T}, w_{i}^{T}=z_{i 0}^{T} A_{12}{ }_{22}\left(\lambda_{10}\right), i=1, \ldots, p$

$$
r_{2}=0\left[\begin{array}{c}
\omega_{10}^{T} \\
\vdots \\
\omega_{p 0}^{T}
\end{array}\right]=0 r
$$

with diw $r=p \times(n-m)$, dim $Q=m \times$ put otherwise arbitrary.

$\checkmark$ can be rewritten into a form which emphasizes the fact that case 3 is inbetween case 1 and case 2. To see this, we assume that $b_{1}$ is cransformad into:

$$
B_{1}=\left[\begin{array}{ll}
A & 0 \\
0 & 0
\end{array}\right]
$$


where $\Lambda$ is a diagonal matrix.

Let $A_{12}, B_{2}$ and $V_{2}$ be partitioned as:

$$
\begin{aligned}
& A_{12}=\left[\begin{array}{l}
A_{121} \\
A_{122}
\end{array}\right] \downarrow \begin{array}{l}
I\left(B_{1}\right) \\
\left\lceil m-r\left(B_{1}\right)\right.
\end{array}
\end{aligned}
$$

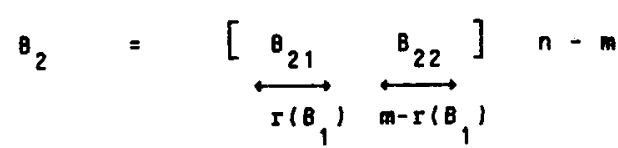

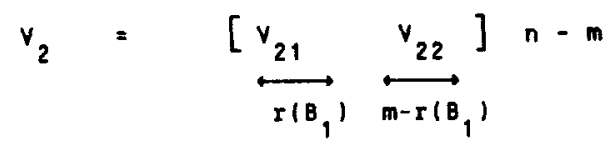

The second condition in $(6-10)$ can now be written as :

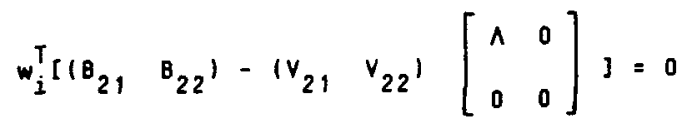$$
i=1, \ldots, n-m
$$

8y selecting $V_{21}$ as:

$$
v_{21}=B_{21} \wedge^{-1}
$$

(6-15) will be reduced to:

$$
w_{i}^{\top} B_{22}=0, i=1, \ldots, n-m
$$

where the left eigenvectors $w_{i}^{\top}$ in $(6-17)$ are given by:

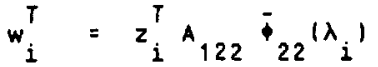

where $\bar{\phi}_{22}\left(\lambda_{i}\right)=\left(I z-\bar{A}_{22}\right)^{-1}$

$$
\text { and } \bar{A}_{22}=A_{22}-B_{21} \wedge{ }^{-1} A_{121}
$$

$w_{i}^{\top} B_{22}=0$ can pow be satisfied for maximally $p$ eligenvectors $w_{i}$ if $\lambda_{i}=\lambda_{i 0}$ in $(6-18)$ are

selected as thé transinissión zeros of

$S\left(A_{22}, B_{22}, A_{122}\right)$, which are equal to the transmission zeros of $S(A, B, C)[6]$.

$$
z_{i 0^{\top}}^{\top}{ }_{122}{ }_{22}^{\left(\lambda_{i 0}\right) B_{22}}=0, i=1, \ldots, p
$$

Now it is straightforward to see that $V_{22}$ is parameterized by:

$$
\begin{aligned}
& v_{22}=-\left[\begin{array}{c}
w_{1}^{T} \\
\vdots \\
w_{n-m}^{T}
\end{array}\right]^{-1}\left[\begin{array}{c}
z_{1}^{T} \\
\vdots \\
z_{n-m}^{T}
\end{array}\right]^{T}=-w^{-1} z \\
& \text { with } z_{i}^{\top}=z_{i 0}^{\top} \\
& w_{i}^{T}=z_{i 0}^{T} A_{122}{ }^{\top}{ }^{\prime}\left(\lambda_{i 0}\right), i=1, \ldots, p
\end{aligned}
$$

The resulting $v_{2}$ is:

$$
\begin{gathered}
v_{2}=\left[B_{21} \Lambda^{-1},-W^{-1} l\right] \\
\text { where }-W^{-1} z \text { is given in }(6-20) \text {. }
\end{gathered}
$$

The remaining $n-m-p$ conditions in $(6-10)$ must be satisfied by selecting $K_{2}$ as before:

$$
k_{2}=0\left[\begin{array}{c}
T \\
w_{10}^{T} \\
\vdots \\
w_{p 0}^{T}
\end{array}\right]=0 r
$$

with $\operatorname{din} r=p \times(n-m)$.

A fow important consequences of exact LTR for minimal-order observers are now discussed here:

* If the system does not have any zeros, exact recovery is still possible with the solution $K_{0}=0, i . e$. no feedback from the state estimates. However in square discrete-time system $r(C B)$ is often maximal, which ensure that $I(B)$ is maximal and that $6(z)$ has the maximum possible number of finite zeros, $p=n-m$. In this special case exact LTR is possible only by selecting the observer gain $V_{\text {. The }}$ feedback gain $K$ is free to choose, and it is possible to use systematic design rules (e.g. LQG-design / for the $K$ selection for stability and loop-shape reqirements. This is a very useful result for LTR design in discrete-time systems because a full-state target design can be recovered, without affecting this original design, simply by choosing the minimal-order observer gain, whereas it is not possible with a full-order observer. Here the full-state design is constrained. Note that by using a minimal-order observer in compensators will require that the processing time of computing the control signal is negligible in comparioson to the sampling interval. The processing time in this case will, however, be reduced compared with the processing time when a filtering observer is used and therefore the minimal-order observer is more attractive than the filtering observer.

* The result in case $3(6-21)$ is the general result for exact recovery with minimal-order observer, since the solution constrains case 1 and 2 as special cases.

* Good input sensitivity and stability robust ness for plant input modelling errors can always be achived if the target $100 p K \$ B$ has full rank. This is only guaranteed if $p>m$ in case 1 and 3 . In case $2 k \notin 8$ has generically full rank, and therefore good feedback properties can be achieved.

* Finally note that dual results for the plant output cannot be invoked, due to the missing duality of minimal-order observers.

\section{MON-MINIMI-PHASE SYSTEMS}

The results for LTR with minimal-order observers of \$ 6 were based on a minimum-phase assumption. If this assumption is not valid some new results can be obtained. In the following the tree usual cases will be discussed independently, but a basic prerequisite will be the recovery conditions.

$$
\begin{aligned}
& K_{2}\left(I z-A_{22}+V_{2} A_{12}\right)^{-1}\left(B_{1}-V_{2} B_{2}\right)=0(7-1) \\
& \sum_{i=1}^{n-n} \quad \frac{K_{2} v_{i} w_{i}^{\top}\left(B_{1}-V_{2} B_{2}\right)}{z-A_{i}}=0
\end{aligned}
$$


where the symbols are defined in $\$ 5,6$.

Further let the number of plant zeros be $p$ and the number of minimum-phase zeros be $q$.

Case 1. $\quad r\left(B_{1}\right)=0$

In this case the recovery condition becomes:

$$
k_{2} v_{i}=0 \text { or } w_{i}^{\top} B_{2}=0, i=1, \ldots, n-m(7-2)
$$

Que to the stability requirements only a subset $q_{T}$ of the possible solutions to the condition $w_{i} B_{i}=0$ can be selected, $i$-. the a solutions:

$$
\begin{aligned}
& z_{10}^{T} A_{12}{ }_{22}\left(A_{10}\right) B_{2}=0 \\
& \left|\lambda_{i 0}\right|<1, i=1, \ldots \ldots, q
\end{aligned}
$$

where $\lambda_{i 0}$ are the zeros of $S(A, B, C)-s e$ [6]. The rearining $n-m-q$ conditions constrains $K$. As in 5 . the solution becomes:

$$
\begin{aligned}
& v_{2}=\left[\begin{array}{c}
w_{1}^{\top} \\
\vdots \\
w_{n-m}^{\top}
\end{array}\right]^{-1}\left[\begin{array}{c}
z_{1}^{\top} \\
\vdots \\
z_{n-m}^{T}
\end{array}\right] \\
& w_{i}^{T}=\sum_{i 0}^{T} A_{12} \phi_{22}\left(\lambda_{10}\right), z_{i}^{T}=z_{i 0}^{T}, i=1, \ldots, q \\
& K_{2}=\bar{g}\left[\begin{array}{c}
T \\
w_{10} \\
\vdots \\
w_{q 0}
\end{array}\right]=\overline{\bar{r}} \bar{r}
\end{aligned}
$$

$\operatorname{din} \overline{\mathbf{a}}=\omega \times \mathbf{q}$

$\overline{\mathbf{g}}$ is a matrix of free parametefs. The rewaining $n-n-q$ pairs $\left(\lambda_{i}, z_{i}\right)$ are free parameters.

Care 2. $\left.\quad{ }_{1} B_{1}\right)=m$

Now the recovery condition becomes

$$
K_{2} v_{i}=0 \text { or } w_{i}^{t}\left(B_{2}-v_{2} B_{1}\right)=0, i=1, \ldots, n-m
$$

As before only a solutions to the conditions $w_{i}^{\top}\left(B_{2}^{\top}-v_{2} B_{2}\right)=0$ can be used, i.e.

$$
\begin{aligned}
& { }_{i 0}^{T}\left(B_{1}+A_{12}{ }_{22}\left(\lambda_{i 0}\right) \theta_{2}\right)=0, i=1, \ldots, q \\
& \left|\lambda_{i 0}\right|<1
\end{aligned}
$$

and $A$ is a zero of $S(A, B, C)$ (see [6] for detaifl).

The reasining $n-m-q$ conditions must be satisfied by slecting $K$ appropritely. The expressions for $V_{2}$ and $K$, are similar to $(7-4)$ with eq. $(7-6)^{2}$ substifuted for eq. $(7-3)$.

$$
\text { Case 3. } \quad 0<r\left(\theta_{1}\right)<m
$$

In this case the recovery condition are as $(7-5)$. The q possible stable solutions to $w_{1}\left(B_{2}-V_{2} B_{1}\right)$ are given by $e q \cdot(7-6)$. The last $n-m-q$ constrains $K_{2}$ - and the expressions for $V$, and $K$, are similar to $q .(7-4)$, with eq. $(7-6)^{2}$ substituted for eq. $(7-3)$.
A general coment for these results concerns the selection of $K_{2}$. In all three cases the matrix $K$ is not free to assign, hence stability-design and loop-shape design are not as straightforward as one would desire. Otherwise the coments from 54 are also valid here. Hotice again that the achievable loop-shapes - subject to the exact recovery constraint - are parameterized explicitly in terms of $K, i$.e. the free parameters $Q$ and the left eigenvectors $w_{i 0}^{f}\left(k_{2}\right)$ and $k_{1}$.

\section{EXnives}

Consider the plant

$$
G(s)=\frac{1 \cdot 2 s}{1 \cdot s} \frac{4}{s^{2}+0.8 s+4} \frac{1}{s}
$$

Let the sampling time be 0.25 sec. The discretetime version $6(2)$ then has zeros at:

$$
z_{1}=0.8825, z_{2}=-0.2502, z_{3}=3.3968
$$

and $G(z)$ is non-ainimum phase. By applying the exact recovery procedure for full-order observers of $\$ 3$ the compensator becomes

$$
H(z)=
$$

$$
\frac{w_{1}\left(z-z_{2}\right)+w_{2}\left(z-z_{1}\right)}{\left(z-z_{1}\right)\left(z-z_{2}\right)}
$$

Where $w_{1}$ and $w_{2}$ are the 2 elements of $q$. The resulting $100 p^{2}$ transfer is then:

$$
K 4 B=6(2) H(2)
$$

$$
=0.019(z+3.3968)\left(\left(w_{1} \bullet w_{2}\right) z-\left(w_{1} z_{2}+w_{2} z_{1}\right)\right) / d
$$

Here denotes the characteristic polynonium of A.

As expected the non-minimum phase zero shows up in $K \gg \theta$. $w_{1}$ and $w_{2}$ are free design paraweters which determines the shape of $K \$ B$ and stability of the closed-100p system. Notice how the performance for the non-minimum phase controlloop is characterized directly by $w_{1}$ and $w_{2}$.

As the second example consider the plant:

$A=\left[\begin{array}{cccr}1.0044 & -5.2447 E-3 & 1.4029 E-3 & 1.4436 E-2 \\ 5.1372 E-5 & 1.0001 & 2.3995 E-1 & -5.6845 E-1 \\ -5.2161 E-5 & 5.5818 E-3 & 9.9980 E-1 & 2.2215 E-2 \\ -1.7897 E-4 & -2.0729 E-4 & -1.2551 E-7 & 9.8419 E-1\end{array}\right]$

$B=\left[\begin{array}{rr}3.5825 E-3 & -8.6189 E-2 \\ 9.9769 E-4 & 2.4174 E-5 \\ -1.4399 E-3 & 1.2011 E-3 \\ -3.4725 E-3 & -0.1575 E-5\end{array}\right] C^{\top}=\left[\begin{array}{cc}1.0 & 0 \\ 0 & 1.0 \\ 0 & 0 \\ 0 & 0\end{array}\right]$

This is an example from [4] transforned into for required for minimal-order observer design. In [4] it was attempted to design a discrete LOG/LTR regulator, but a finite recovery error was obtained for all frequences. Here a minimalorder observer will be applied. The system is minimum-phase with zeros at $t+0.99982$, 0.99668 ). The sampling-time is $0.01 \mathrm{sec}$.

A target feedback design is given by:

$K=\left[\begin{array}{rrrr}3.3072 E+2 & 1.8503 E+3 & 2.2942 E+4 & -9.2927 E+3 \\ -1.0656 E+3 & -4.2362 E+3 & -7.3194 E+4 & 2.8251 E+4\end{array}\right]$ 
A nominal observer is designed as $v_{2}=-w^{-1} z$ with eigenvalues at $(5.32 \mathrm{E}-3,-1.8 \mathrm{E}-\mathrm{l})$. $A$ recovery trajectory is defined from $V$, to the exact LTR value $V_{2}=B_{3} B^{-}$by moving the rom the nominal to the LTR-values as functions of $a$, so that

$$
\lambda_{i}(q=0)=\lambda_{i 0}, \lambda_{i}(q+\infty)=\lambda_{i, L T R}
$$

and equally for $z_{i}^{\top}$. And $V_{2}(q+-)=B_{2} \theta_{1}^{-1}$.

The plot of the singular values of the full loop transfer is shown in fig. 1 and 2 for different values of $q$. Clearly recovery is achieved. The final value of $v_{2}$ which achives exact recovery is:

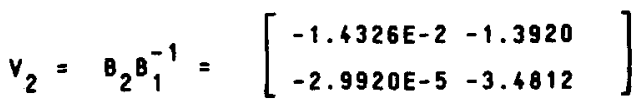

\section{REFEIEIEES} feedback design:Concepts for a classical/modern synthesis" IEEE Transact. Aut. Control, AC-26,1,P.6-16,1981.

[2] H. Kwakernaak and R. Silvan: "Linear optimal control systems " Hiley Interscience, N.Y. USA, 1972.

[3] 6. Stein and M. Athans: "The L06/LTR procedure for multivariable feedback control design". IEEE Transact. Aut. Control, AC-32, 2,p.105-114,1987.

[4] 6. C. Goodman: "The L06/LTR method and discrete-time control systems". M.Sc. thesis, rep.no. LIDS-TH-1392, Lab of Inf. and Dec. Syst. MIT,MA,USA, 1984.

[5] M. Athans: 'A tutorial on the LOG/LTR method". Proc. American Control Conf., Seattle, WA,p.1289-96,1986.

[6] P. Segaard-Andersen: "Issues in robust multivariable observer-based feedback design". Ph.D thesis, Control Engineering Inst. Technical University of Denmark, 1986.

[7] E. J. Davison and S. H. Hang: "On pole assignment in linear nultivariable systens using output feedback", IEEE Transact. Aut, Control, AC-20,p.516-8, 1975.

[8] U. Shaked and $N$. Karcanias: - The use of zeros and zero-directions in modelreduction". Int. J. Control,23,1,p.11335,1976 .

(9] A. N. Andry jr.,E. Y. Shapiro and J. C. Chung: Eigenstructure assignment in linear systems" IEEE Transact. on Aerospace and Electr. Syst. AES-19,5, p. 711-29, 1983.

[10] P. Swgaard-Andersen: "Explicit solution to the problem of exact loop transfer recovery". Proc. American Control Conf p150-151, Minneapolis, MN, 1987.

(11) J. M. Maciejowski: Asymptotic recovery for discrete-time systons", IEEE

Transact. Aut. Control, $A C-30,6, p .602-5$, 1985.

[12] J. S.Freudenberg and 6. P. Looze:

- Right half plane poles and zeros and design trade-offs in feedback systems". IEEE Transact. Aut. Control. $A C-30,6, p .555-65,1985$

[13] K. J. Astrom, P. Hagander and J. Sternby "Zeros of sampled systems", Automatica, $20,1, p .31-8,1984$.

[14] C. E. Rohrs, 6.Stein and K. J. Astrom: "Uncertainty in sampled systems". Proc. American Control Conf.,p.95-7,1985.

[15] R. Isermann: "Oigital Control systems". Springer-Verlag, Berlin 1981.

[16] P. Sfgaard-Andersen "Loop transfer recovery with minimal-order observers" Proc. IEeE Conf. on Decision and Control Los Angeles, CA,p.933-938, 1987 .

[17] J. R. Dowdle: -Robust observer-based compensators". Ph.D. thesis MIT, Cambridge, HA 1979.

[16] Z. Zhang and J.S. Freudenberg: "Loop transfer recovery with non-minimum phase zeros" Proc. IEEE Conf. on Decision and Control, Los Angeles, CA,p.956-957,1987.
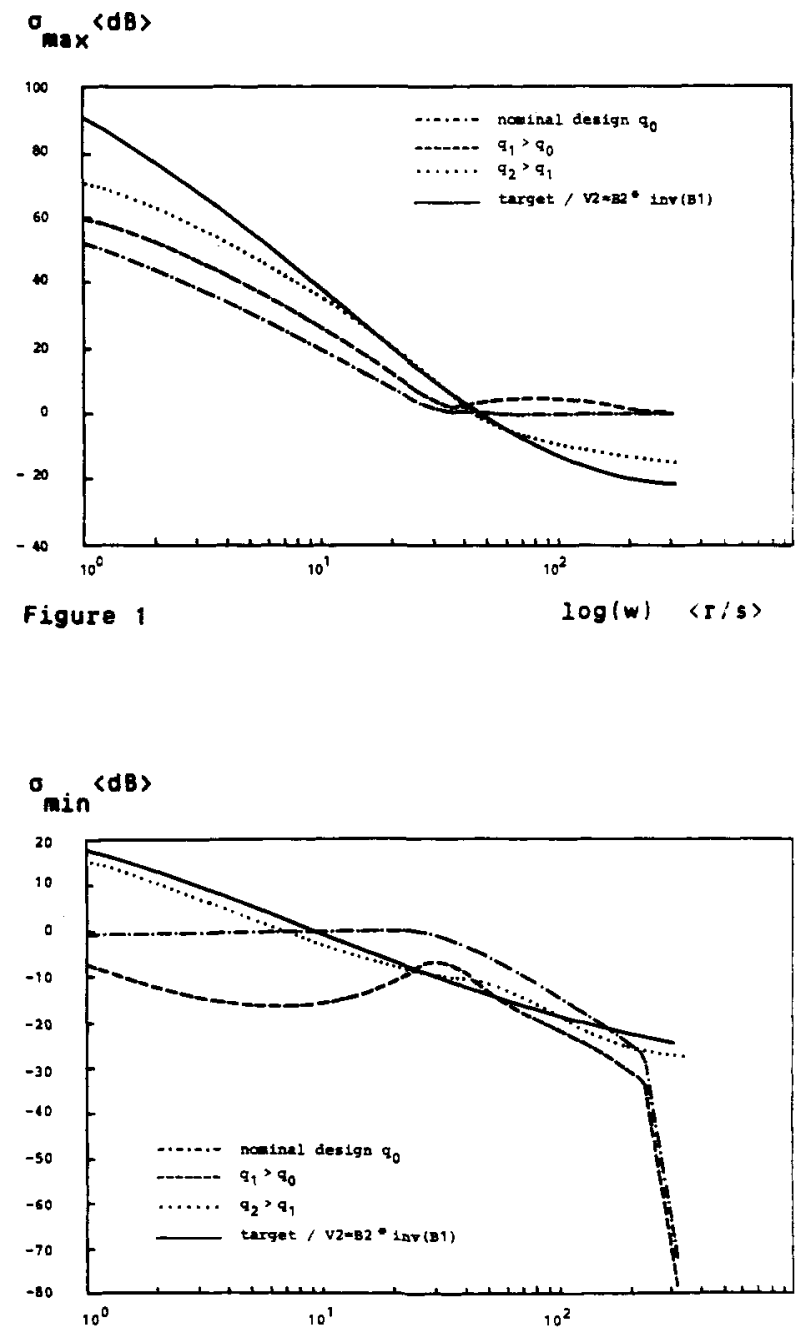

Figure 2

$\log (w)\langle r / s\rangle$ 